\title{
TOWARDS CONTEXTUAL GOAL-ORIENTED PERCEPTION FOR PEDESTRIAN SIMULATION
}

\author{
Laure Bourgois ${ }^{1}$, Julien Saunier ${ }^{1}$ and Jean-Michel Auberlet ${ }^{1}$ \\ ${ }^{1}$ Université Paris Est, ISFTTAR-IM-LEPSIS, 58 Bld Lefèbvre, 75015 Paris, France \\ \{laure.bourgois, julien.saunier, jean-michel.auberlet\}@ifsttar.fr
}

\begin{abstract}
Keywords: Simulation, Evaluation, Perception
Abstract: $\quad$ Perception is often seen in multiagent systems and in robotics from a passive point of view. The sensors of the agent collect information on its environment; however the potentially important number of percepts is not realistic and may decrease the agents efficiency. In this article, we introduce a contextual goal-oriented perception filtering. Besides the lack of plausibility of omniscient agents, it addresses the problem of transmitting too much information to the agents. This goal-oriented perception module is evaluated model in terms of validity of the resulting behavior and of time complexity.
\end{abstract}

\section{INTRODUCTION}

The use of traffic simulation tools to design and assess the infrastructure is widespread for highways or interurban networks. However, several issues remain to apply it realistically, in urban areas, because the environment is more complex. In particular, pedestrians are difficult to take into account, since their behavior is less constrained and normative than the behavior of the vehicles. Current pedestrian simulation tools do not consider heterogeneity and dynamics of the environment sufficiently, especially for road crossing or sidewalk following. This paper describes a pedestrian model that takes into account the interaction with other pedestrians (on sidewalks) and vehicles (for road crossing). This model is based on perception operations which are inputs of decision and action components.

Perception is often seen in multiagent systems from a passive point of view. The sensors of the agent collect information on its environment; however the potentially important number of percepts is not realistic and may decrease the agents efficiency. Moreover, an agent does not always need a complete information about its environment. The complexity of simulation models and the amount of information clearly leads to a bottleneck for the simulation of thousands of pedestrians.

The perception can be seen as a three-part process (Weyns et al., 2004): an agent senses the state of the environment to get a raw perception, then it interprets the raw perception to transform the data into per- cepts (consistent with its internal representation of the world), and finally it filters these percepts to keep only relevant percepts (filtered perception). In this framework, we propose a refinement of the classical perception model that improves the filtering to keep only the relevant information. The filters are goal-driven and depend on the current task of the agent. They also take into account the context of the agent. Besides the lack of plausibility of omniscient agents, the problem of transmitting too many information to the agents is that of scalability. Accessing and treating this information is combinatorial in function of the number of agents. Henceforth, many simulation tools include $a d$ hoc ways to limit the perception, but to the best of our knowledge they are rarely evaluated.

In section 2, a non exhaustive state of the art on microscopic pedestrian models is presented, stressing on the computational features of perception and navigation space representation. Section 3 presents our pedestrian model, which enable the agents to move in urban areas thanks to two different models (one for moving and the other for decision making).Section 4 deals with the navigation model. In this case, the field of vision depends on the agent dynamic context. We present our model and assess both the validity of the model and its computation cost. Section 5 presents the road-crossing decision model. Here, the percept filtering depends both on the context (traffic fluidity) and on the agent's goal (crossing decision). We show some results of our simulations in terms of treatment complexity gains. To conclude, we present our works outlook. 


\section{RELATED WORKS}

The Social Force Model (SFM) (Helbing and Molnar, 1995 ) is the most used in crowd simulation community. A pedestrian agent acts as if it was subject to attractive forces (its destination ...) and repulsive forces (pedestrians ...). It tries to trail straight ahead in order to reach its destination following a shortest path route, and avoiding obstacles. Helbing interprets the private sphere notion as "repulsive sustained field" sum computed in function of environment obstacles and an attractive field function of a wanted walking speed and a given destination (and possible "attractive" pedestrians to form groups (Moussaïd et al., 2010)). This model has several limitations; due to its reactive feature, a pedestrian agent avoids obstacles at the last moment, moreover it has an unlimited depth of the field of vision that leads to a perception complexity in $O\left(n^{2}\right)$ (with $n$ the number of pedestrians). Hoogendoorn's model (Hoogendoorn and Bovy, 2000) partially takes up SFM and adds control theory concepts to it. A pedestrian agent optimizes an utility function with a set of different costs for acceleration, private space sphere and path diversion. In graphical computer community, Reciprocal Velocity Obstacle model (Guy et al., 2010) is widely used and shows the advantage of avoiding the classical phenomenon of oscillation between pedestrian (major shortcoming of SFM model). This model takes into account for a pedestrian agent the reaction time and the altering pedestrian speed time. Authors code pedestrian agents trajectory as a linear programming problem. As the SFM, these models use a continuous space representation with at least a $O\left(n^{2}\right)$ complexity ( $n$ the number of pedestrians) (Hoogendoorn and Bovy, 2000).

However, some other environment modelings exist with different shape cells division (Blue and Adler, 2001) or data structures. These cells enable to geometrically characterize a set of zone perceived by the pedestrian agents. Their major advantage lies in the decreasing of the perception complexity. Indeed, the complexity of Reynolds initial method is $O\left(n^{2}\right)$ for boids modeling ( $n$ the number of boids); it falls to $O(k n)$ with a discrete space ( $k$ the number of cells) (Reynolds, 2000). Nevertheless, this approach has a certain time computation cost, merely, it is executed off line. The method has the major shortcoming of a constant perception modeling, whereas a pedestrian does not necessarily pay attention to every nearby zone with the same intensity.

Collision avoidance is generally based on the perception in order to detect moving and/or fixed obstacles (other pedestrians, vehicles, walls). Although perception has long been considered as an undergone state, it has emerged recently that awareness is an active state, and not only the result of stimuli. The perception is directed towards a subject via a receptor.

The detection of the obstacles is executed at each time step. Therefore, in order to simulate several thousands of agents, the perception process should be implemented with a low complexity algorithm. We choose to characterize the complexity of the interaction between agents as the number of perceptual operations that an agent must perform to interact with another agent, whereas we consider that the agent's decision time and the agent's decision execution time are constant.

\section{PEDESTRIAN AGENT MODEL}

As our model does not depend on a space representation, we consider in this article a continuous space (the worst case in term of complexity). Besides, a continuous-space model is well suited for simulating real pedestrians and allows to knows for each step the exact position of each agents. Moreover, we focus on the inputs of perception and decision components, hence we do not go into details regarding action algorithms (more details in (Bourgois et al., 2010)).

We use the simplifying assumption that the pedestrians do not try to form groups. We do not focus on the lane formation self organization.

Our model consists of two models, one for moving and one for road-crossing decision. In this section, we will first present the general characteristics of an agent then we will detail each model.

\subsection{Pedestrian agent characteristics}

Following Helbing recent model (Johansson et al., 2007), a pedestrian is represented by a circle with a variable radius. Each agent has a set of variables and parameters $P$ for the pedestrian interactions that mainly are : its obstacle reaction intensity $A$ and a reactivity threshold $B$, over which the agent does not react anymore to environmental elements.

We add here $I$ the set of characteristics necessary to model pedestrian-vehicle interactions: a vehicle perceptual field, that is to say the depth of the field of vision on the infrastructure (e.g. the street) and a crossing willingness, which depends on the environmental context perceived by the agent.

\subsection{Models involved}

We follow Hoogendoorn's model concepts (Hoogendoorn and Bovy, 2000) that distinguish 3 decision levels: strategic, tactical, and operational. We assume 
that the route of an agent (its origin and destination) is already known (strategic level). The model is therefore composed of: a moving model for obstacle avoidance (operational level) and a decision model for road crossing (tactical level).

The moving model takes place within the framework of perception-action, and the decision model lies in the perception-decision framework. To ensure the continuity of pedestrian movement (along a sidewalk, pedestrian crossing), we must articulate these two models. If an agent always tries to avoid all the obstacles, the priority of an agent crossing a street is above all to avoid vehicles. When the agent decides to stop at the curb to allow vehicles to pass, it does not apply the moving model.

We briefly detail the behavior of an agent according to its position (sidewalk / road) and its state. For each situation, we distinguish two main functions: final destination computation and navigation. The following Navigation algorithm is given here:

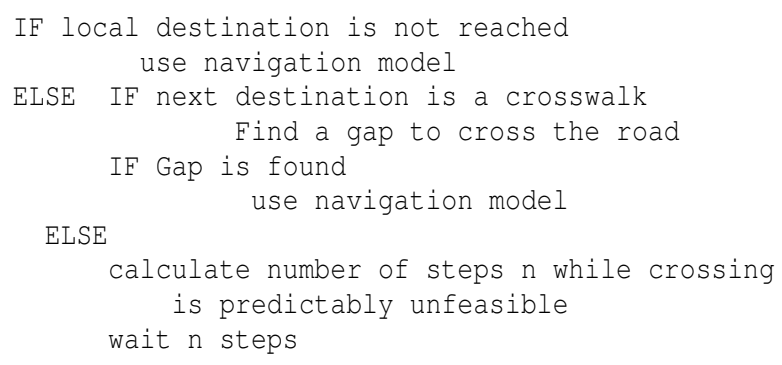

Once an agent reaches a sidewalk, it computes its local destination ${ }^{1}$.

\section{SHORT-TERM NAVIGATION}

\subsection{Moving model (operational level)}

Perception is based on the depth of the field of vision and the sensibility parameter (that determines the anisotropy of the agent). In the initial perception model, an agent $\alpha$ may be influenced by the agent $\beta$, if is in the field of vision of agent $\alpha$. This influence is represented by a force exerted on $\alpha$ by $\beta$. This force depends on the sensibility of $\alpha$ to $\beta$ and on $g\left(d_{\alpha \beta}\right)$ the distance dependant reaction of agent $\alpha$ to $\beta$ which is defined as :

$$
g\left(d_{\alpha \beta}\right)=A_{\alpha} e^{\frac{R_{\alpha}+R_{\beta}-d_{\alpha \beta}}{B_{\alpha}}}
$$

where $R_{\alpha}, R_{\beta}$ are the radii of the agents and $d_{\alpha \beta}$ is the distance between their centers.

We see that the force decreases when the distance between the pedestrians increases. We remark here

\footnotetext{
${ }^{1}$ To avoid artificial pedestrian convergence on the same geometrical destination, an agent randomly calculates its own local destination corresponding to an area surrounding the given local destination.
}

that perception does not take into account the speed nor the course of the agents $\alpha$ and $\beta$, but only their respective locations. Helbing did not mention the depth of the field of vision, we thus added a variable in order to perceive only the neighbors. We calibrated $A$ and $B$ with the same value as recent SFM.

In the initial moving model, each agent has a desired speed, and tries to walk in a (desired) direction, which depends on its current position and its destination. It also tries to maintain its speed to its desired speed, which is therefore the target for the agent to reach. Agent acceleration is then defined by: its actual speed, its impatience and the repulsive forces to avoid obstacles (pedestrians, walls) and to keep its private sphere.

In previous works, we enhanced this model (Bourgois et al., 2010) with sensitivity measures and by introducing a depth of the field of vision for pedestrianpedestrian interactions. We have proved that the depth of the field of vision influences the pedestrians' flows. In those research, we tested a depth that is a good trade-off collision avoidance and speed preservation.

The depth of the field decreases the number of perception operations. This perception efficiency improvement comes from the filtering of the perception.

We now add a dynamic adaptation of the depth of the field of perception to acquire only the relevant data depending on the agent context.

Relevance distance We compute the depth of the field of vision depending on the density of agents in the neighborhood of the agent. Indeed, we observed in our simulations that the high density of agents tends to reduce the agent acceleration to zero. Based on the intuitive idea that in congestion situations, a pedestrian perceives only pedestrians nearby, we propose to modify the depth of the field of vision in function of the density of agents surrounding the agent.

To reproduce this phenomenon, we introduce a filtering function that allows relevant agent selection according to the density of agents. This function consists in a distance computation from which the neighbors are taken into account for the acceleration computation. In this way, if the number of neighbors is low, the agent takes into account distant neighbors, while if the density is large, it only considers the nearest neighbors. The following filtering function has been determined empirically:

$$
D_{p}=\left(e^{\frac{1}{n b_{\text {neighbor }}}}-1\right) * \alpha
$$

with $D_{p}$ the relevance distance, $n b_{\text {neighbor }}$ the raw number of neighbors perceived by the agent and $\alpha$ a factor which has been calibrated to $10 \times e$.

Thanks to this formula, the agent relevance distance variates according to the number of pedestrians present in its field of perception. We initialize the pa- 
rameters thanks to the results found in our previous study : the depth of the field of vision ranges from $1,65 m$ to $9 m$.

From the relevance distance, we compute theoretically the maximum number of agents that could be present (i.e. treated) surrounding the given agent for that distance. It is the relevant-distance area divided by the maximum surface occupied by one pedestrian (even though the maximum possible density, reported to be $5.4 \mathrm{ped} / \mathrm{m}^{2}$ (Weidmann, 1993) is use, the number of perceived pedestrian is bounded). After a threshold (15), the field of vision becomes too little to contain more than 12 agents. This result can be explained by the underlying formula: when there are few agents in the system, the field of vision is wide, hence enabling the agents to perceive all the other agents. However, the field of vision diminishes when the number of agents increases.

The operational complexity of perception is $O(k n)$ where $n$ is the number of simulated agents and $k$ the maximum number of agents that can fit in the neighborhood of an agent. We now compare these theoretical computations to empirical results.

\subsection{Experimentation and results}

In order to validate and verify the consistency of our model, we executed several series of simulations. For these simulations, we have recreated the standard conditions described in (Lobjois and Cavallo, 2009).

The next sections present our simulation results in order to quantify the validity and efficiency of the perception function. In the first case, we simulated a bidirectional corridor, which is similar to a sidewalk: pedestrians enter the corridor at one end and leave it at the other end. The size of the corridor is $10 \mathrm{~m} \times 60 \mathrm{~m}$. The flow (ped/h) is the number of agents created at each end of the corridor.

Perceptual improvements For each simulation of bidirectional corridor, we recorded the average intensity difference between the force sustained with a raw perception and the one with a filtered perception. In this way, we measure the difference in behavior induced by the filtering process. We see in Figure 1 that for high flows, the pedestrian acceleration force with a filtered perception is weaker than the one with a raw perception; although the maximum difference is $0.4 \mathrm{dm} / \mathrm{s}^{2}$ (at worst a $3 \%$ average speed deviation). Therefore, the behaviors are not or slightly modified by the active perception. A second validation aims to compare our simulation with a global behavior of pedestrian flows. For that aim, we have aggregated the individual data and compared with the existing data like fundamental diagrams (FD) (which describe

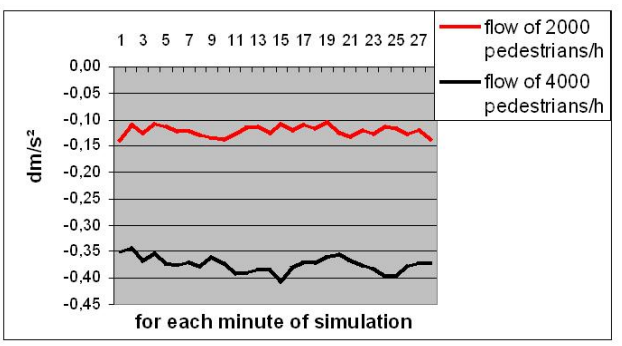

Figure 1: Average intensity difference (2000, $4000 \mathrm{ped} / \mathrm{h})$.

the relation between mean speed, density and flow of pedestrians).

Unfortunately, literature concerning pedestrian FD is poor, mainly because of the difficulty to acquire data. Our experimental results shows that our model approximates correctly other aggregate measures found in the literature (Weidmann, 1993).

Improvements in bidirectional corridors Figures 2 present for each minute of simulation, the records for all agents, of: the average and maximum number of agents perceived (raw perception) and the average and maximum number of agents treated (filtered perception).

For a low flow, the average number of agents perceived and treated are comparable, the difference between the maximum number of perceived and treated agents is negligible. Indeed, with a low density, the relevance distance is always close to the maximal distance of perception $(9 \mathrm{~m})$. The efficiency gain is marginal but always positive.

The more the flow increases (Figure 2), the more the difference between raw and filtered perception increases. For 2000 agents, the filtered perception treats only $30 \%$ of the raw perception. In the previous sec-

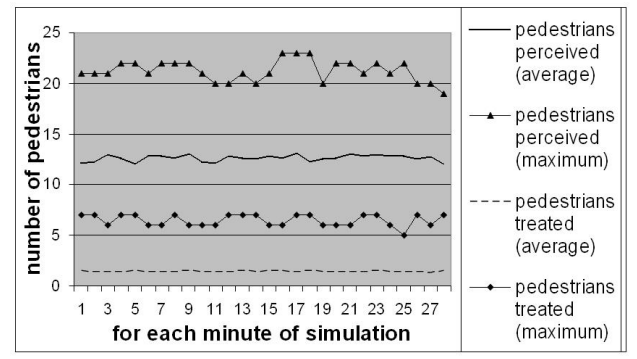

Figure 2: Flow of $2000 \mathrm{ped} / \mathrm{h}$.

tion, we showed that the filtering function did not deteriorate noticeably the behavior of the agents. The simulation results show that the filtering function is very efficient in mid to high flows and that the number of percepts an agent has to treat decreases strongly $(-70$ to $-90 \%)$. 


\section{ROAD CROSSING}

\subsection{Decision model (tactical level)}

This model involves two models: Tom's model (Auberlet et al., 2011) and a "gap acceptance" model (acceptance of time slot for road crossing).

Tom's model is based on the environment perception. It establishes a set of Boolean variables, each corresponding to a visual cue used cross. The model allows to predict with a reliability close to $70 \%$ the decision in these contexts. We interpret the output of this model as a crossing willingness This value modulates a "gap acceptance" model (the pedestrian decides to cross if the time slot offered by the traffic is higher than its own estimate of the crossing time).

For the decision model, the agent gets one or several vehicle queues moving along the road. The agent selects the first vehicle that can either let it pass through or block it. Other vehicles are not treated. This filter is thus based on a prediction : the time to collision (TTC) between the pedestrian and the vehicle, if all agents maintain their actual speeds. We point out this filter is different from the relevance filter (based on the actual situation, the density of agents). This prediction is based on the assumption that one can anticipate the movement of perceived vehicles (a standard procedure in agent-oriented or distributed simulation).

We distinguish two types of context for vehicles perception: free traffic flow and congested traffic flow. In case of congested traffic flow, the agent avoids the vehicles on the roadway as fixed obstacles. In this situation, the agent only perceives the stationary vehicles between which it wants to get through. Hence, the perception depends on the current goal of the agent and of its context (free or congested traffic). In a free traffic flow context, if the agent raw perception is an upstream queue of vehicles, it selects only the vehicle with which it might interact.

The perception and decision functions must take into account: "side time": time taken by the pedestrian to reach the vehicle's side (in function of the distance between the side of the vehicle and the pedestrian), "lag before" (or front lag) (time for the vehicle front bumper to reach the side of the pedestrian), "lag behind" (time for the vehicle rear bumper to be completely beyond the pedestrian).

We notice that if the agent is waiting on the curb, it does not longer need to perceive. We can compute the time it has to wait before deciding to cross again with the no perception condition.

WHILE Lag behind < Side time
wait without perception
This function decrease the computation time because the agent no longer has to perceive and to treat information while it is stopped.

\subsection{Tactical perception complexity}

Each time an agent decides to cross, it must perceive a set of lanes and for each a set of vehicles. The number of perceived vehicles depends on: the number of lanes, $m$ and the field of perception, implying a maximum number of vehicles perceived, $k$.

Taking into account exceptional situations with a very high traffic density and assuming that there is no perception filter, the maximum number of perceived vehicles for a crossing decision is $O(m k)$.

\subsection{Results}

The vehicles are modeled as agents which have a basic car following task behavior. We use Ketenci's work (Ketenci et al., 2010) to animate these agents. However, here we do not focus on the vehicle coordination task on roads intersections. Currently, the vehicle agents only perceive each other.

In order to study plausible situations, we initialized the parameters with values found in the literature (Lobjois and Cavallo, 2009). The vehicle agents are created with the following features: Time Headway (THW) between the vehicles, distributed according to the normal law on $[1,2, \ldots, 8] s$, speed is distributed uniformly on the intervals [38-42], [48 - 52], and $[58-62] \mathrm{km} / \mathrm{h}$. We simulated the road crossing of a $285 m$ long unidirectional road (Staplin, 1995). We simulate two different vehicle flows: 400 and 600 vehicles per hour.

Behavior validity For each simulation, we recorded for each decision taken by an agent: the speed of the selected interacting vehicle and the TTC (in seconds). Indeed, referring authors take into account an initiation crossing time (the pedestrian start to move on the sidewalk toward the road whereas there is still a vehicle in front of him). Actually, a pedestrian take also into account the time of arrival of the upstream vehicle, i.e., the TTC.

We observed that the mean values of TTCs belong to the following range of values : $[5.02,7.8]$. In our simulations, the crossing behavior is consistent with the time slots observed. Indeed, for the time slots taken by the agents, more than $95 \%$ of pedestrians would cross in the reality.

Improvements in street crossing situations We recorded for each agent: the number of decision(s) condition it has to make to cross the road, with the possibility to block the perception (no perception con- 
dition) when the agents knows it will not be able to cross because of an incoming vehicle, the number of decision(s), no_condition it would have to make without the no perception condition.

Table 1 presents the average gain of perceptiondecision cycles with the no perception condition : it is the difference between the average of condition and no_condition for all the agents. We see an important gain, even for high speeds. It is not surprising that this gain also increases with a higher flow, since the agent has less opportunities to cross. The road-

Table 1: Average gain of decision depending on the average vehicle speed.

\begin{tabular}{|l|c|c|c|}
\hline Gain Vehicle speed & 30 & 40 & 50 \\
\hline \hline Flow of 400 veh/h & 6,79 & 5,56 & 5,67 \\
Flow of $600 \mathrm{veh} / \mathrm{h}$ & 15,62 & 11,88 & 9,25 \\
\hline
\end{tabular}

crossing model diminishes the number of treatments, both in terms of road-crossing decisions and of number of vehicles perceived, while keeping acceptable behavior results.

\section{CONCLUSION}

We have presented a pedestrian agent model encompassing both action (locomotion) and decision (street crossing). Both tasks are highly dependent on the perception of the situation, but do not rely on the same pieces of information. Hence, we have shown that the refinement of the perception, based on a goal-oriented filtering of the percepts, enables the decrease of the number of objects treated by the agents while keeping consistent results in term of behavior.

The difference between the raw perception and the filtered perception has been evaluated and depends on the model: from $70 \%$ to $90 \%$ for the locomotion model. The filtering we have proposed is not an implementation artefact to ameliorate the time complexity of our agents, but a part of the human cognitive process. We note, that humans can only acknowledge and handle a limited amount of information. Future works needs to be done to establish which percepts must be kept to ameliorate the perception process.

More research is needed to better understand and reproduce a group behavior (Moussaïd et al., 2010), on particular in the street crossing case. Donikian notes that groups show bolder behavior by accepting shorter time gaps and by forcing the vehicle drivers to slow down (Donikian, 2004). Several problems arise (group identification and formation). Another interesting possibility to model large areas would be to switch from a microscopic to a macroscopic model at run-time.

\section{REFERENCES}

Auberlet, J.-M., Bodard, V., Bourgois, L., Brémond, R., Désiré, L., Maestracci, M., Granié, M.-A., Rabier, R., and Tom, A. (2011). Simulation de traversée de carrefour par des piétons. Technical report, Fondation Sécurité Routière, projet SiCAP. (to be published).

Blue, V. J. and Adler, J. (2001). Cellular automata microsimulation for modeling bi-directional pedestrian walkways. Transportation Research Journal Part B.

Bourgois, L., Oulhaci, A., and Auberlet, J.-M. (2010). Simulation de déplacement de piétons : Vers un modèle de perception et de prédiction d'action chez autrui. In Journées Francophones des Systèmes Multi-Agent.

Donikian, S. (2004). Modélisation, contrôle et animation d'agents virtuels autonomes évoluant dans des environnements informés et structurés. Habilitation Diriger des Recherches, Institut de Recherche en Informatique et Systmes Alatoires.

Guy, S. J., Lin, M. C., and Manocha, D. (2010). Modeling collision avoidance behavior for virutal humans. In 9th International Conference on Autonomous Agents and Multi-agent Systems.

Helbing, D. and Molnar, P. (1995). Social force model for pedestrian dynamics. Physical Review E.

Hoogendoorn, S. and Bovy, P. (2000). Gas-kinetic modeling and simulation of pedestrian flows. Transportation Research Record.

Johansson, A., Helbing, D., and Shukla, P. (2007). Specification of the social force pedestrian model by evolutionary adjustment to video tracking data. Advances in Complex Systems.

Ketenci, U., Brémond, R., Auberlet, J.-M., and Griselin, E. (2010). Bounded active perception. In Proceedings EUMAS, European Workshop on Multi-agent Systems.

Lobjois, R. and Cavallo, V. (2009). The effects of aging on street-crossing behavior: From estimation to actual crossing. Accident Analysis and Prevention.

Moussaïd, M., N.Perozo, Garnier, S., Helbing, D., and Theraulaz, G. (2010). The walking behaviour of pedestrian social groups and its impact on crowd dynamics. In PLOS ONE.

Reynolds, C. (2000). Interaction with groups of autonomous characters. In Game Developers Conference.

Staplin, L. (1995). Simulator and field measures of driver age difference in left-turn gap judgments. Transport Research Record.

Weidmann, U. (1993). Transporttechnik der fussgänger, transporttechnische eigenschaften des fussgngerverkehrs (literturauswertung)). Technical report, Schriftenreihe des IVT Nr. 90, Zweite, ergnzte Auflage, Zrich.

Weyns, D., Steegmans, E., and Holvoet, T. (2004). Towards active perception in situated multiagent systems. Special Issue of Journal on Applied Artificial Intelligence. 